\title{
The European Journal of Case Reports in Internal Medicine The First Two Years
}

\author{
John G Kellett \\ Editor in Chief of the European Journal of Case Reports in Internal Medicine
}

Published: 03/05/2016

How to cite this article: Kellett GJ. The european journal of case reports in internal medicine - the first two years. EJCRIM

2016;3:doi:10.12890/2016_000438

Conflicts of Interests: The Author declare that there are no competing interests.

This article is licensed under a Commons Attribution Non-Commercial 4.0 License

\section{EDITORIAL}

The European Journal of Case Reports in Internal Medicine (EJCRIM) is just over two years old. To date 400 reports have been submitted and just under half have been accepted for publication. In order to keep costs down our initial policy was to charge a small fee for all submissions, and a further small publication fee for papers that were accepted. However, we have now abolished the submission charge and replaced it with a single, slightly increased, publication charge for all accepted papers.

All journals find getting papers peer reviewed difficult. Some reviewers respond quickly but may not be thorough, others respond slowly but are meticulous, while others are both slow and sloppy. Worse, reviewers may have diametrically opposed opinions. After a few months experience it became clear to EJCRIM's editors that submissions fell into three categories - those reports that were clearly suitable for publication, those that could be quickly rejected, and only a small minority that required more careful scrutiny before a decision could be reached. If we were going to continue to send every paper out for multiple reviews we realized that this would considerably delay the publication process of excellent submissions, as well as prolonging the agony for the authors of rejected papers. We would also soon exhaust the good will of all our volunteer reviewers. We have, therefore, changed our editorial policy. All submissions are now first read by the Editor-in-Chief and then discussed weekly by the Editorial Board. At this meeting papers are rejected, accepted with or without minor revisions, or sent to an associate editor for review. In addition we have devised a new template for authors to use (Table 1).

The biggest single reason for rejecting a paper is that there is no message or learning point. Before submitting a case report authors should really think very carefully about why the report is important and of interest. What is another doctor going to learn from it? Many authors delude themselves into thinking that a report of something that has never been seen before is a major discovery. On the contrary it is patently a very, very minor discovery. What might be a major discovery, however, is something important that has never been noticed before. This, for example, could be a rare presentation of a common condition, especially if it lead to a serious diagnostic or therapeutic error.

All too often we receive a report of something that no one has ever seen before, and probably no one will ever see again, and the learning point is that every doctor should now actively look for it in every patient. It would be far better if a plausible argument was made for why something important might not have been noticed before, accompanied by a realistic discussion of the clinical implications together with practical advice on what, if anything, to do about it in everyday practice. Furthermore, EJCRIM would encourage reports of small clusters of cases, rather than a single case, that supports the argument for something important that might not have been noticed before.

Currently the big important things in medicine that nobody might have noticed before are likely to be in the following areas:

1. Incidental findings: increasingly findings are being discovered during diagnostic investigations, which reveal diagnoses that were previously considered to be rare but are now becoming common place. For example, pituitary adenomas are now commonly detected, and parathyroid disease diagnosed more frequently and with greater certainty. 
2. Overlooked clinical findings: it is obvious that many of the cases submitted to EJCRIM were examples where obvious historical and physical examination findings were initially overlooked and only eventually recognised after exhaustive expensive investigations. Doctors are making these mistakes all the time, but either not recognizing them or admitting to them. The most common cause of a delayed or misdiagnoses is a diagnostic "blind spot," such as a conscious decision not to pursue a clinical finding, failure to account for a symptom or sign, atypical presentations and/or inadequate follow-up of abnormal laboratory results. Moreover, the most common medical errors are failures to obtain a proper medical history, order the appropriate diagnostic tests, and provide adequate follow up ${ }^{[1]}$. EJCRIM would welcome frank accounts, with well defined and argued learning points, of such honest yet expensive mistakes. It is obviously highly educational and valuable to remind every doctor that they should listen to and examine every patient, and provide pertinent examples of what happens when they forget to.

3. New treatment side effects and complications: whilst of interest to EJCRIM, authors should not forget that they have a responsibility to report adverse medication events to the relevant post-marketing drug surveillance authorities.

The other big reason for submission rejection is lack of research. It could be argued that the biggest single advance in medicine over the last 20 years is the easy access to unlimited up to date information. The days of travelling to the nearest medical school library and turning page after page of Index Medicus in search of an elusive paper are over. Thanks to the internet and its search engines an exhaustive review of the world literature now only takes a few seconds and, in many cases, entire papers are instantly available without charge. Despite these advances we often find, after a few moments on Google, that many authors have hardly performed any background literature searches at all. We realise that limiting the number of references may have discouraged authors from referencing all the articles they researched and have, therefore, changing our policy on this issue. From now on we invite authors to provide the results of any of their literature searches as an appendix to their submission.

Papers that are likely to require detailed and longer review are those that report a truly esoteric issue that requires unusual expertise, or that are highly controversial or make highly unlikely or even incredible claims. Another common issue is whether or not the paper is appropriate for publication in an internal medicine journal, and would not be better placed in a sub-speciality or other type of journal.

There are a number of other minor irritants that may put the Editor and his reviewers in a bad mood: too many authors, too many words, too much repetition and too much detail. If a report is to be interesting to read then less is definitely more. Authors should strive to keep their papers within 1000 words. This is especially important when English is not your first language - the shorter your report the less mistakes made and the more easily they are corrected. Ideally the learning point or message of the report should be clear from the title, the abstract, the introduction, the case itself and the discussion.

At the present time EJCRIM is not listed in MEDLINE. The reason for this, we are told, is that our reports of not of sufficient quality. We hope that this will be improved by the adherence of our submitting authors to our new template. However, in the final analysis, the quality of what we publish depends on what is submitted. Currently it costs less to publish a case report in EJCRIM than any other journal. We look forward to continued improvement in our journal for both our authors and readers in the years ahead. 


\section{MESSAGE}

What is the message (Learning points): Please select from the list below and please add your explanatory paragraph in the text field.

a. Novelty (i.e. how it will change the practice of medicine)

$$
\begin{array}{ll}
\text { a. } & \text { Knowledge - new facts or new insights into old facts } \\
\text { b. Technology - new or new use for old } \\
\text { c. Treatment - new }
\end{array}
$$

b. Instructive errors -
a. "Wrong footing" errors - errors of initial impression
b. Rare presentation of a common condition
c. Errors of Diagnostic strategy
d. Incorrect information
e. Missing information
f. Over investigation
g. Under investigation
h. Misleading investigations
i. Inappropriate investigations
j. Failure to detect serious illness or deterioration

c. System errors
a. Ergonomics
b. Healthcare complexity
c. Communication

d Human errors
a. Clinical judgement and/or Experience
b. Cognitive pitfalls
c. Diagnostic errors
d. Management errors

\section{CASE REPORT}

\section{DISCUSSION:}

a. what's known already

b. what's new

c. why is this case/s significant

\section{REFERENCE LIST (Where can I find out more?)}

a. References

b. Literature search-appendix

\section{REFERENCES}

1. McDonald CL, Hernandez MB, Gofman Y, Suchecki S, Schreier W.The five most common misdiagnoses: a meta-analysis of autopsy and malpractice data. The Internet Journal of Family Practice 2008; 7. 